\title{
Exercise in Waist-High Warm Water Decreases Pain and Improves Health-Related Quality of Life and Strength in the Lower Extremities in Women With Fibromyalgia
}

\author{
N. GUSI, ${ }^{1}$ P. TOMAS-CARUS, ${ }^{1}$ A. HÄKKINEN,${ }^{2}$ K. HÄKKINEN, ${ }^{3}$ AND A. ORTEGA-ALONSO ${ }^{4}$
}

Objective. To evaluate the short- and long-term efficacy of exercise therapy in a warm, waist-high pool in women with fibromyalgia.

Methods. Thirty-four women (mean \pm SD tender points $17 \pm 1$ ) were randomly assigned to either an exercise group (n = 17) to perform 3 weekly sessions of training including aerobic, proprioceptive, and strengthening exercises during 12 weeks, or to a control group $(n=17)$. Maximal unilateral isokinetic strength was measured in the knee extensors and flexors in concentric and eccentric actions at $60 \%$ second and $210 \%$ second, and in the shoulder abductors and adductors in concentric contractions. Health-related quality of life (HRQOL) was assessed using the EQ-5D questionnaire; pain was assessed on a visual analog scale. All were measured at baseline, posttreatment, and after 6 months.

Results. The strength of the knee extensors in concentric actions increased by $20 \%$ in both limbs after the training period, and these improvements were maintained after the de-training period in the exercise group. The strength of other muscle actions measured did not change. HRQOL improved by $93 \%(P=0.007)$ and pain was reduced by $29 \%(P=0.012)$ in the exercise group during the training, but pain returned close to the pretraining level during the subsequent de-training. However, there were no changes in the control group during the entire period.

Conclusion. The therapy relieved pain and improved HRQOL and muscle strength in the lower limbs at low velocity in patients with initial low muscle strength and high number of tender points. Most of these improvements were maintained long term.

KEYWORDS. Fibromyalgia; Muscle strength; Pain; Quality of life; Exercise.

\section{INTRODUCTION}

Patients with fibromyalgia (FM) have muscle weakness (1), widespread muscle pain, and lower pressure pain thresholds of tender sites in the upper and lower limbs $(2,3)$. In addition to pain, the most prominent symptoms in FM are

Supported by the European Social Funds and Regional Covernment of Extremadura (Spain; grant 2PR02B017 and Health Department).

${ }^{1}$ N. Gusi, PhD, P. Tomas-Carus, PhD: Fitness and Lifequality Laboratory, University of Extremadura, Cáceres, Spain ${ }^{2}$ A. Häkkinen, PhD: Jyväskylä Central Hospital, Jyväskylä, A. Häkkinen, PhD: Jyväskylä Central Hospital, Jyväskylä,
Finland; ${ }^{3} \mathrm{~K}$. Häkkinen, PhD: University of Jyväskylä, JyväsFinland; 'K. Häkkinen, PhD: University of Jyväskylä, Jyväskylä, Finland; ${ }^{4}$ A. Ortega-Alonso, MSc: Fitness and Lifequality Laboratory, University of Extremadura, Cáceres, Spain, and University of Jyväskylä, Jyväskylä, Finland.

Address correspondence to $\mathbf{N}$. Gusi, PhD, Sports Sciences Faculty, University of Extremadura, Avenue Universidad s/n, 10071 Cáceres, Spain. E-mail: ngusi@unex.es.

Submitted for publication June 17, 2005; accepted in revised form August 26, 2005. localized to peripheral soft tissues, and there have been numerous studies of abnormalities in muscle structure and function in individuals with FM. The muscle tissues of these individuals are reportedly characterized by ultrastructural abnormalities and DNA fragmentation not related to apoptosis, aging, or deconditioning (4).

Muscle strength depends on both the cross-sectional area of the muscle and the neural activity. The crosssectional area of the quadriceps femoris muscle has been reported to be within normal ranges in persons with FM $(5,6)$. However, some studies have shown that maxima isometric and dynamic muscle strength in short tests (1-5 repetitions) is lower in persons with FM than in healthy individuals $(1,5,7,8)$; however, these reports have been contradicted in the longer tests that are more related to resistance $(9,10)$. The reduced maximal muscle strength of knee extensors has been associated with lower outcomes in functional tests related to daily living (sit up and sit down on a chair, walking) in persons with FM (11) and with the increase of disability, especially in females with 